\title{
Improve Self-Adaptive Control Parameters in Differential Evolution for Solving Constrained Engineering Optimization Problems*
}

\author{
Tam BUI** ${ }^{* *}$, Hieu PHAM ${ }^{* *}$ and Hiroshi HASEGAWA** \\ ** College of Systems Engineering and Science, Shibaura Institute of Technology \\ 307 Fukasaku, Minuma-ku, Saitama-shi, Saitama 337-8570, Japan \\ E-mail: nb12509@shibaura-it.ac.jp, pnh112@gmail.com, h-hase@shibaura-it.ac.jp
}

\begin{abstract}
We proposed a new improvement of self-adaptive strategy for controlling parameters in differential evolution algorithm (ISADE). The differential evolution (DE) algorithm has been used in many practical cases and has demonstrated good convergence properties. It has only a few control parameters as number of particles $(N P)$, scaling factor $(F)$ and crossover control $(C R)$, which are kept fixed throughout the entire evolutionary process. However, these control parameters are very sensitive to the setting of the control parameters based on their experiments. The value of control parameters depend on the characteristics of each objective function, so we have to tune their value in each problem that mean it will take too long time to perform. We present a new version of the DE algorithm for obtaining self-adaptive control parameter settings that show good performance on numerical benchmark problems and constrained engineering optimization problems.
\end{abstract}

Key words : Differential Evolution (DE), Global-Local Search, Multi-Peak Problems

\section{Introduction}

Differential evolution is an optimization technique originally proposed by Storn and Price (1). In DE, new individuals are generated by mutation and crossover operator, which uses the variance within the population to guide the choice of new search points. Although DE is very powerful, it may sometime fall into local optimum and has a slow convergence speed in the last period of iterations. The aim of this work is to improve self-adaptive differential evolution, to do this the three DE's mutation scheme operators are selected as candidates due to their good performance on problems with different characteristics. These three mutation scheme operators are chosen to be applied to individuals in the current population with the same probability. The scaling factor $F$ is calculated by ranking the population and applying formula of sigmoid function depend on the rank number of population size and the crossover control $C R$ is also adaptively changed instead of taking fixed values to deal with different classes of problems. Another critical parameter of DE, the population size NP remains a userspecified variable to tackle problems with different complexity. The results from experiment show that our algorithm with improve self-adaptive control parameter settings is better than or at least comparable to the standard DE algorithm and evolutionary algorithms (EAs) from literature when considering the quality of the solutions obtained and calculation cost. All the algorithms are applied to the some benchmark functions and compared based on some different metrics.

This paper is organized in the following manner. The section 2 gives a briefly introduce to the DE and related work of DE. Section 3 describes ISADE. Section 4 evaluates the performance of ISADE on the some benchmark test functions and apply the new ISADE for solving some constrained engineering optimization problems. Finally, a few conclusions are given in

*Received 19 Dec., 2012 (No. 12-0510) [DOI: 10.1299/jcst.7.59]

Copyright $\subset 2013$ by JSME 
Section 5.

\section{Review of DE and related work}

\subsection{Formulation of the optimization problem}

The optimization problem is formulated in this section. The design variable, objective function and constraint condition are defined as follows:

Design variable: $x=\left[x_{1}, \ldots, x_{D}\right]$

Objective function: $f(x) \rightarrow$ Minimum

Modified objective function: $f^{*}(x)=f(x)+\gamma P(x) \rightarrow$ Minimum

Inequality constraint functions: $g_{j}(x) \leq 0, j=1, \ldots, m$

Equality constraint functions: $h_{k}(x)=0, k=1, \ldots, n$

Range of design variables: $x_{i}^{l b} \leq x_{i} \leq x_{i}^{u b}$

where $f(x), \gamma$ and $f^{*}(x)$ denote objective function, penalty coefficient and modified objective function, respectively. $x^{l b}=\left[x_{1}^{l b}, \ldots, x_{D}^{l b}\right], x^{u b}=\left[x_{1}^{u b}, \ldots, x_{D}^{u b}\right]$, and $D$ denote the lower boundary condition vectors, upper boundary condition vectors, and number of design variable vectors, respectively. $m$ and $n$ are the number of inequality and equality constraints respectively. $g_{j}$ and $h_{k}$ are linear or nonlinear real-value functions respectively.

\subsection{Differential evolution}

Differential evolution (DE), proposed by Storn and Price ${ }^{(1)}$, is a very popular EA. Like other EAs, DE is a population-based stochastic search technique. It uses mutation, crossover and selection operators at each generation to move its population toward the global optimum minimum.

2.2.1. Initialization in DE The initial population was generated uniformly at random in the range lower boundary $(L B)$ and upper boundary $(U B)$.

$$
X_{i j}^{G=0}=l b_{j}+\operatorname{rand}_{j}(0,1) *\left(u b_{j}-l b_{j}\right) \quad \operatorname{rand}_{j}(0,1) \text { a random number in }[0,1] .
$$

2.2.2. Mutation operation In this process, $\mathrm{DE}$ creates a mutant vector $V_{i}^{G}=\left(V_{i, 1}^{G}, \cdots\right.$, $V_{i, D}^{G}$ ) for each individual at each generation $G, X_{i}^{G}$ (called a target vector) in the current population.

There are several variants of DE, according to ${ }^{(1),(2)}$ we have some mutation schemes as follow:

$$
\begin{aligned}
& \text { DE/rand/1: } V_{i, j}^{G}=X_{r_{1}, j}^{G}+F *\left(X_{r_{2}, j}^{G}-X_{r_{3}, j}^{G}\right) \\
& \text { DE/best/1: } V_{i, j}^{G}=X_{\text {best }, j}^{G}+F *\left(X_{r_{1}, j}^{G}-X_{r_{2}, j}^{G}\right) \\
& \text { DE/current to best/1: } V_{i, j}^{G}=X_{i, j}^{G}+F *\left(X_{b e s t, j}-X_{i, j}^{G}\right)+F *\left(X_{r_{1}, j}^{G}-X_{r_{2}, j}^{G}\right) \\
& \text { DE/rand/2: } V_{i, j}^{G}=X_{r_{1}, j}^{G}+F *\left(X_{r_{2}, j}^{G}-X_{r_{3}, j}^{G}\right)+F *\left(X_{r_{4}, j}^{G}-X_{r_{5}, j}^{G}\right) \\
& \text { DE/best/2: } V_{i, j}^{G}=X_{b e s t, j}^{G}+F *\left(X_{r_{1}, j}^{G}-X_{r_{2}, j}^{G}\right)+F *\left(X_{r_{3}, j}^{G}-X_{r_{4}, j}^{G}\right) \\
& \text { DE/rand to best/1: } V_{i, j}^{G}=X_{r_{1}, j}^{G}+F *\left(X_{b e s t, j}-X_{r_{1}, j}^{G}\right)+F *\left(X_{r_{2}, j}^{G}-X_{r_{3}, j}^{G}\right)
\end{aligned}
$$

where $r_{1}, r_{2}, r_{3}, r_{4}$ and $r_{5}$ are distinct integers that randomly selected from the range [1,NP] and are also different from $i$. The parameter $F$ is called the scaling factor that amplifies the difference vectors. $X_{\text {best }}$ is the best individual in the current population. 
2.2.3. Crossover operation After mutation process, DE performs a binomial crossover operator on $X_{i}^{G}$ and $V_{i}^{G}$ to generate a trial vector $U_{i}^{G}=\left(U_{i, 1}^{G}, \cdots, U_{i, D}^{G}\right)$ for each particle $i$ as shown in (Eq.14).

$$
U_{i}^{G}= \begin{cases}V_{i, j}^{G} & \text { if } \operatorname{rand}_{j}(0,1) \leq C R \text { or } j=j_{\text {rand }} \\ X_{i, j}^{G} & \text { otherwise. }\end{cases}
$$

where $i=1, \cdots, N P, j=1, \cdots, D, j_{\text {rand }}$ is a randomly chosen integer in $[1, D], \operatorname{rand}_{j}(0,1)$ is a uniformly distributed random number between 0 and 1 generated for each $j$ and $C R \in[0,1]$ is called the crossover control parameter. Due to the use of $j_{\text {rand }}$, the trial vector $U_{i}^{G} \operatorname{differs}$ from target vector $X_{i}^{G}$.

2.2.4. Selection operation The selection operator is performed to select the better one between the target vector $X_{i}^{G}$ and the trial vector $U_{i}^{G}$ to enter the next generation.

$$
X_{i}^{G+1}= \begin{cases}U_{i}^{G} & \text { if } f\left(U_{i}^{G}\right) \leq f\left(X_{i}^{G}\right) \\ X_{i}^{G} & \text { otherwise. }\end{cases}
$$

where $i=1, \cdots, N P, X_{i}^{G+1}$ is target vector in the next population.

\subsubsection{DE algorithm pseudo-code $\quad$ Require: $N P, C R$ and $F$.}

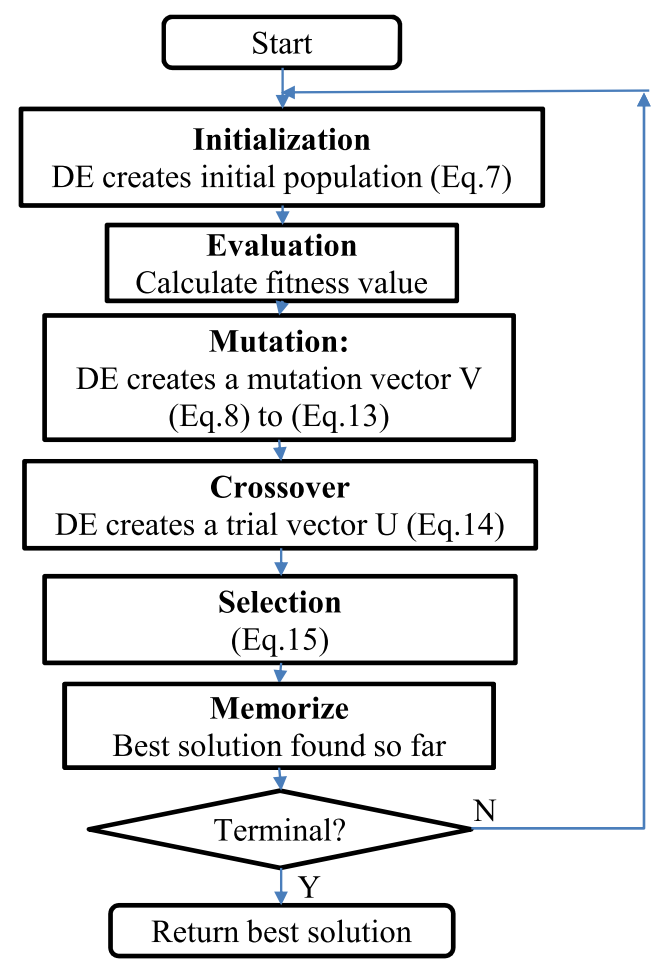

Fig. 1 Procedure of DE.

\subsection{Related work of DE}

This section reviews some papers that compared the different extension of DE with the original DE. After that, we concentrate on papers that deal with parameter control in DE.

There have been many research works on controlling search parameters of DE. DE Control parameters include the $N P, F$ and $C R$.

Storn and Price ${ }^{(1)}$ argued that these three control parameters are not difficult to set for obtaining good performance. They suggested that $N P$ should be between $5 D$ and $10 D, F$ should be 0.5 as a good initial choice and the value of $F$ smaller than 0.4 or larger than 1.0 will lead to performance degradation and $C R$ can be set to 0.1 or 0.9 . 
Omar S.Soliman and Lam T.Bui at ${ }^{(3)}$, the author introduced a self-adaptive approach to DE parameters using a variable step length generated by a Gaussian distribution; also, the mutation amplification and crossover parameter were introduced. These parameters are evolved during the optimization process.

A.K.Qin and P.N.Suganthan ${ }^{(4)}$ proposed the new choice of learning strategy SaDE and the two control parameters $F$ and $C R$ do not require predetermining. During evolution, suitable learning strategy and parameter are applied. Here, author proposed learning strategy adaptation is to probabilistically select one out of several available learning strategies and apply to the current population. The reason for author's choice is that these two strategies have been commonly used in many DE literatures and reported to perform well on problems with distinct characteristics. Among them, "rand/1/bin" strategy usually demonstrates good diversity while the "current to best/2/bin" strategy shows good convergence property, two candidate strategies, assuming that the probability of applying strategy "rand/l/bin" to each individual in the current population is $p 1$, the probability of applying another strategy should be $P 2=1-p 1$. After specified number of generations called the "learning period".Then, the probability of $p 1$ is updated. The author considered allowing $F$ to take different random values in the range $(0,2]$ with normal distributions of mean 0.5 and standard deviation 0.3 for different individuals in the current population. For $C R$ author assumed $C R$ normally distributed in a range of normal distribution of $C R(C R m)$ and standard deviation 0.1 . The CR values associated with trial vectors successfully entering the next generation are recorded. After a specified number of generations $C R$ has been changed for several times under the same normal distribution with center $C R m$ and standard deviation 0.1 , and author recalculated the $C R m$ according to all the recorded $C R$ values corresponding to successful trial vectors during this period.

J.Liu and J.Lampinen ${ }^{(13)}$ present an algorithm based on the Fuzzy Logic Control (FLC) in which the step-length was controlled using a single FLC. Its two inputs were: linearly depressed parameter vector change and function value change over the whole population members between the current generation and the last generation.

$\mathrm{J}_{\text {.Teo }}{ }^{(16)}$ proposed an attempt to dynamic self-adaptive populations in differential evolution, in addition to self-adapting crossover and mutation rates, they showed that DE with self-adaptive populations produced highly competitive results compared to a conventional DE algorithm with static populations.

J.Brest ${ }^{(6)}$ presented another variant of DE algorithms jDE, which uses different selfadaptive mechanisms applied on the control parameters: The step length $F$ and crossover rate $C R$ are produce factors $F$ and $C R$ in a new parent vector.

$$
\begin{aligned}
F_{i}^{G+1} & = \begin{cases}F_{l}+\operatorname{rand}_{1} * F_{u} & \text { if } \text { rand }_{2} \leq \tau_{1} \\
F_{i}^{G} & \text { otherwise. }\end{cases} \\
C R_{i}^{G+1} & = \begin{cases}\operatorname{rand}_{3} & \text { if } \text { rand }_{4} \leq \tau_{2} \\
C R_{i}^{G} & \text { otherwise. }\end{cases}
\end{aligned}
$$

where $\operatorname{rand}_{1}, \operatorname{rand}_{2}, \operatorname{rand}_{3}, \operatorname{rand}_{4}$ are uniform random values $\in[0,1] . \tau_{1}$ and $\tau_{2}$ represent probabilities to adjust factors $F$ and $C R$, respectively. Author set $\tau_{1}=\tau_{2}=0.1$. Because $F_{l}=0.1$ and $F_{u}=0.9$, the new takes a value form [0.1,0.9] in a random manner. The new $C R$ takes a value from $[0,1] . F_{i}^{G+1}$ and $C R_{i}^{G+1}$ are obtained before the mutation process.

Through reviewing related work, we understood that it is difficult to select DE learning strategies in the mutation operator and DE control parameters. To overcome this drawback we proposed the Improvement of Self-Adapting control parameters in Differential Evolution (ISADE) - a new version of DE in this research. The detail of ISADE is presented in the next section.

\section{Improvement of Self-Adapting Control Parameters in Differential Evolution}

To achieve good performance on a specific problem by using the original DE algorithm, 
we need to try all available (usually 6 mentions above) learning strategies in the mutation operator and fine-tune the corresponding critical control parameters $C R, F$ and NP. From the experiment we know that the performance of the original DE algorithm is highly dependent on the strategies and parameter settings. Although we may find the most suitable strategy and the corresponding control parameters for a specific problem, it may require a huge amount of computation time. Also, during different evolution stages, different strategies and corresponding parameter settings with different global and local search capability might be preferred. Therefore, to overcome this drawback, we attempt to develop a new version of DE algorithm that can automatically adapt the learning strategies and the parameters settings during evolution. The main ideas of the ISADE algorithm are summarized below.

\subsection{Adaptive selection learning strategies in the mutation operator}

ISADE probabilistically selects one out of several available learning strategies in the mutation operator for each individual in the current population. Hence, we should have several candidate learning strategies available to be chosen and also we need to develop a procedure to determine the probability of applying each learning strategy. In this research, we select three learning strategies in the mutation operator as candidates: "DE/best/1/bin", "DE/best/2/bin" and "DE/rand to best/1/bin" that are respectively expressed as:

$$
\begin{aligned}
& \text { DE/best/1: } V_{i, j}^{G}=X_{b e s t, j}^{G}+F *\left(X_{r_{1}, j}^{G}-X_{r_{2}, j}^{G}\right) \\
& \text { DE/best/2: } \quad V_{i, j}^{G}=X_{b e s t, j}^{G}+F *\left(X_{r_{1}, j}^{G}-X_{r_{2}, j}^{G}\right)+F *\left(X_{r_{3}, j}^{G}-X_{r_{4}, j}^{G}\right) \\
& \text { DE/rand to best/1: } V_{i, j}^{G}=X_{r_{1}, j}^{G}+F *\left(X_{\text {best }, j}-X_{r_{1}, j}^{G}\right)+F *\left(X_{r_{2}, j}^{G}-X_{r_{3}, j}^{G}\right)
\end{aligned}
$$

The reason for our choice is that these three strategies have been commonly used in many DE literatures and reported to perform well on problems with distinct characteristics ${ }^{(1),(2)}$. Among them, "DE/rand to best/1/bin" strategy usually demonstrates good diversity while the "DE/best/1/bin" and "DE/best/2/bin" strategy show good convergence property, which we also observe in our trial experiments.

Since here we have three candidate strategies, the probability of applying strategy to each particle in the current population is $p_{i}$ which are same value $p_{1}=p_{2}=p_{3}=1 / 3$. With this learning strategies in the mutation operator, the procedure can gradually evolve the most suitable learning strategy at different learning stages for the problem under consideration.

\subsection{Adaptive scaling factor $\boldsymbol{F}$ and crossover control parameter $\boldsymbol{C R}$}

3.2.1. Adaptive scaling factor In the multi-point search of the DE, particles move from their current points to new search points in the design space of design variables. For example, as shown in Fig. 2, the particle A requires a slight change to the values of the design variables to obtain the global optimum solution. On the other hand, particle B cannot reach a global optimum solution without a significant change, and in addition, particle $\mathrm{C}$ has landed in a local optimum solution. Such a situation, in which the good individual and the low individual are intermingled, can generally occur at any time in this search process. Therefore, we have to recognize each individual's situation and propose a suitable design variables generation process for each individual's situation in the design space.

In the DE algorithm, the distance for a search point can be changed by controlling the $F$ factor for determining the neighborhood range. To do this, S.Tooyama and H.Hasegawa ${ }^{(17)}$ proposed APGA/VNC approach in which author used sigmoid function to control neighborhood parameter. In this paper, we will sort all the particles by estimating their fitness. A ranked particle is labeled with this rank number and assigned $F$ that corresponds with this number. The formula for $F$ by sigmoid function as follows.

$$
F_{i}=\frac{1}{1+\exp \left(\alpha * \frac{i-\frac{N P}{2}}{N P}\right)}
$$




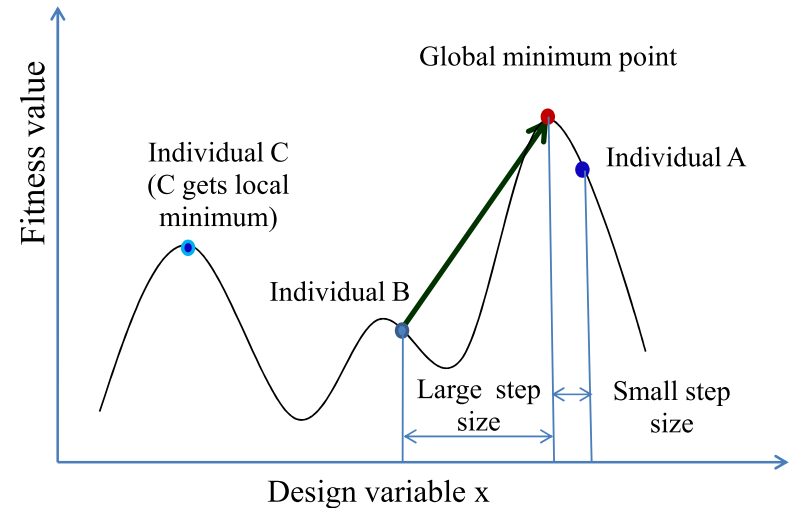

Fig. 2 Example of individual situations.

where $\alpha, i$ denote the gain of the sigmoid function, particle of $i^{t h}$ in $N P$, respectively.

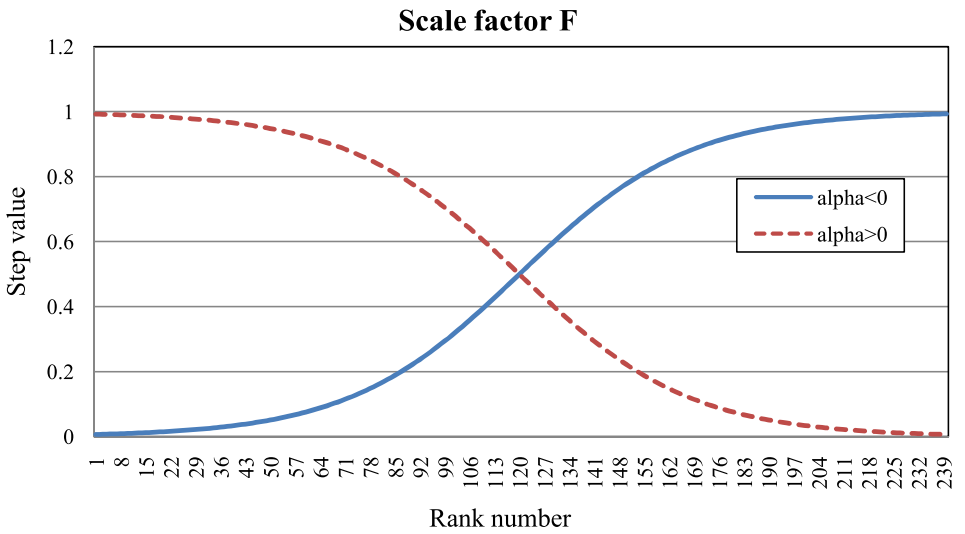

Fig. 3 Suggested to calculate $F$ value.

The gait of $F$ chart depends on the sign and gain of $\alpha$ Fig. 3. When particle at good fitness same as particle A in Fig. 2 will have small step size of $F$ factor and otherwise. From this view, the ISADE method automatically adapts $F$ factor to obtain design variable generation accuracy for each individual's situation and particle's fitness. As a result, we believe that it will steadily provide a global optimum solution and reduce the calculation cost.

For better performance of ISADE it is need that the scale factor $F$ should be high in the beginning to have much exploration and after certain generation $F$ is need to be small for proper exploitation. To implement this, we have new approach to calculate the the scale factor $F$ as follow:

$$
F_{\text {iter }}^{\text {mean }}=F_{\text {min }}+\left(F_{\text {max }}-F_{\text {min }}\right)\left(\frac{\text { iter }_{\text {max }}-\text { iter }}{\text { iter }_{\text {max }}}\right)^{n_{\text {iter }}}
$$

where $F_{\max }, F_{\min }$, iter, $_{\text {iter }}$ max and $n_{\text {iter }}$ denote the lower boundary condition of the $F$, and the upper boundary condition of the $F$, maximum generation, current generation and nonlinear modulation index, respectively. From our experiment we assign $F_{\min }=0.15$, and $F_{\max }=$ 1.55 .

To control the $F_{i t e r}^{\text {mean }}$, we have varied the nonlinear modulation index $n_{\text {iter }}$ with generation as follows:

$$
n_{\text {iter }}=n_{\min }+\left(n_{\max }-n_{\min }\right)\left(\frac{\text { iter }}{\text { iter }_{\max }}\right)
$$

where $n_{\max }$ and $n_{\min }$ are typically chosen in the range $(0,15]$. After a number of experiments on the values of $n_{\max }$ and $n_{\min }$, we have found that the best choice for them is 0.2 and 6.0. The 


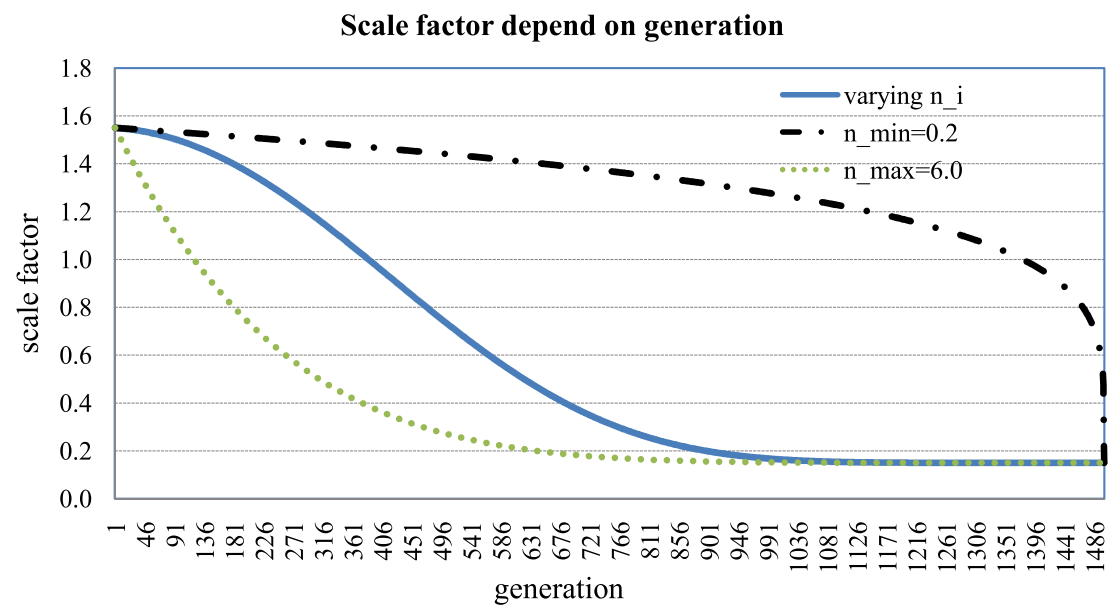

Fig. 4 The scale factor depend on generation.

gait of $F_{\text {iter }}^{\text {mean }}$ chart depends on the iteration number and the nonlinear modulation index $n_{\text {iter }}$ is shown in Fig. 4.

We introduced a novel approach of scale factor $F_{i}$ of the each particles with their fitness values in (Eq.21). So in one generation the value of $F_{i}^{i t e r}(i=1, \cdots, N P)$ are not the same for all particles in the population rather it is made to vary for all particles in each generation. Consider $F_{\text {iter }}^{\text {mean }}$ of (Eq.22) as an average value that we assign to each generation and the final value of scale factor for each particle in each generation is calculated as follow:

$$
F_{\text {iter }}^{i}=\frac{F_{i}+F_{\text {iter }}^{\text {mean }}}{2}
$$

where iter $^{2} 1, \ldots$, iter $_{\text {max }}$ and $i=1, \ldots, N P$

3.2.2. Crossover control parameter G.Reynoso-Meza ${ }^{(15)}$ suggested to have a success if a child substitutes its parent in the next generation. The minimum, maximum and medium value on such set of success is used for this purpose.

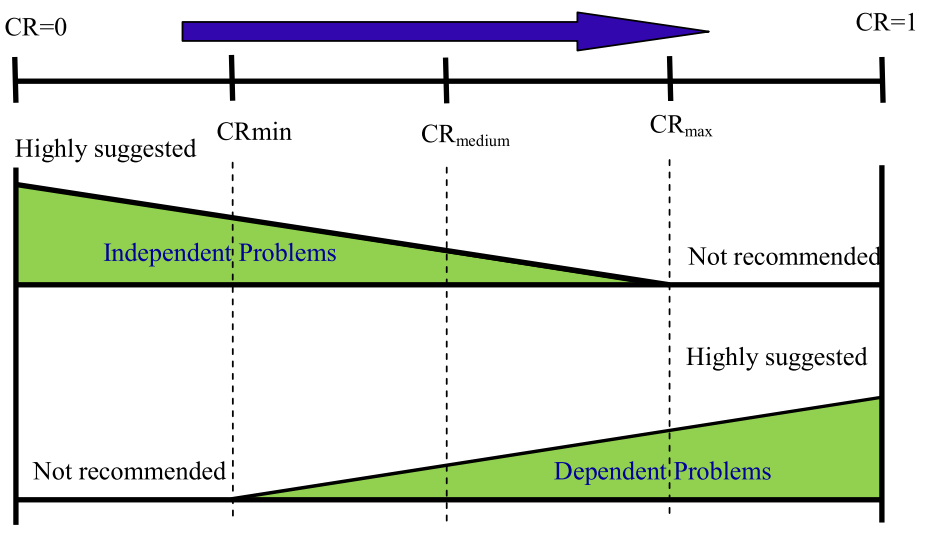

Fig. 5 Suggested to calculate $C R$ values.

- Be able to detect a separable problem, choosing a binomial crossover operator with low values for $C R$.

- Be able to detect non-separable problems, choosing a binomial crossover operator with high values for $C R$.

In this way, the algorithm will be able to detect if high values of $C R$ are useful and furthermore, if a rotationally invariant crossover is required. A minimum base for $C R$ around its median value is incorporated to avoid stagnation around a single value, Fig. 5 shows this principle, so we propose the ideas behind this adaptive mechanism for the crossover: 
The control parameter $C R$ is adapted as follows:

$C R_{i}^{G+1}= \begin{cases}\operatorname{rand}_{2} & \text { if } \operatorname{rand}_{1} \leq \tau \\ C R_{i}^{G} & \text { otherwise. }\end{cases}$

where: $\operatorname{rand}_{1}$ and $\operatorname{rand}_{2}$ are uniform random values $\in[0,1], \tau$ represents probabilities to adjust $C R$, same as ${ }^{(6)}$ we assign $\tau=0.10$.

After that we adjust $C R$ as follows:

$$
C R_{i}^{G+1}= \begin{cases}C R_{\text {min }} & \text { if } C R_{\text {min }} \leq C R_{i}^{G+1} \leq C R_{\text {medium }} \\ C R_{\text {max }} & \text { if } C R_{\text {medium }} \leq C R_{i}^{G+1} \leq C R_{\text {max }} .\end{cases}
$$

where: $C R_{\text {min }}, C R_{\text {medium }}$ and $C R_{\text {max }}$ denote the low value, median value and high value of crossover parameter respectively. From our experiment in many trials, we assign $C R_{\min }=$ $0.05, C R_{\text {medium }}=0.50$ and $C R_{\max }=0.95$.

The purpose of our approach is that user does not need to tune the good values for $F$ and $C R$, which are problem dependent. The rules for improve self-adapting control parameters are quite simple, therefore the new version of the DE algorithm does not increase the time complexity in comparison to the original DE algorithm.

3.2.3. ISADE algorithm pseudo-code Require: $N P$

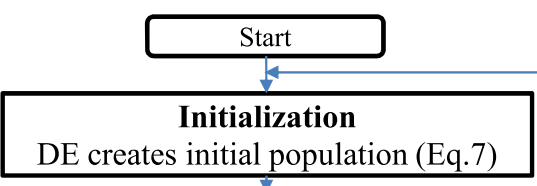

Evaluation rank pupolation

Calculate fitness value and sort population by their descent fitness

Adaptive scaling factor $\mathbf{F}$ (Eq.21) to (Eq.24)

Adaptive crossover control parameter CR (Eq.25) to (Eq.26)

\section{Mutation:}

Apply adaptive selection learning strategies creates a mutation vector $\mathrm{V}$ in (Eq.18) to (Eq.20)

Crossover

DE creates a trial vector (Eq.14)

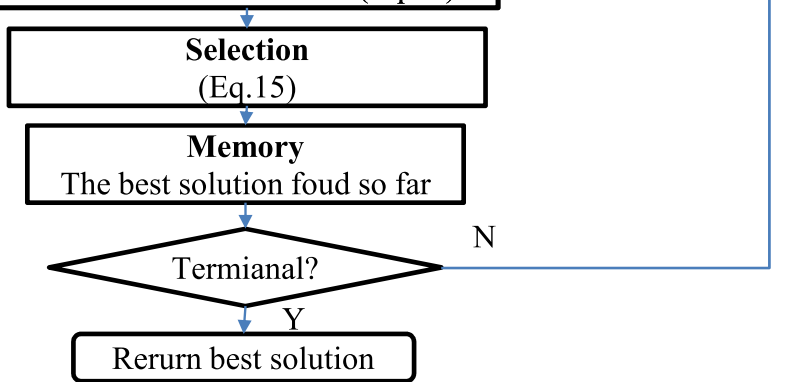

Fig. 6 Procedure of ISADE.

\section{Experiments}

In this section, the first experiment is to be test for turning the $\alpha$ parameter ISADE, after that we will test the robustness of the ISADE method compare with some reference methods as: $\mathrm{jDE}^{(6)}, \mathrm{PSO}^{(18)}$ and $\mathrm{SaDE}^{(4)}$, the last experiment, we will apply ISADE to some constrained engineering optimizations. These experiments are performed 20 trials for every function. 


\subsection{Benchmark functions}

To estimate the stability and convergence to the optimal solution of ISADE, We will use 9 well-known benchmark test functions with 30 dimensions such as Sphere (Sp), Rosenbrock (Ro), Ridge (Ri), Griewank (Gr), Rastrigin (Ra), Ackley (Ac), Levy (Le), Schawefel (Sc) and Alpine (Al) function. These functions are given as follows:

$$
\begin{aligned}
& S p: f_{1}=\sum_{i=1}^{n}\left\{x_{i}^{2}\right\} \\
& R o: f_{2}=\sum_{i=1}^{n}\left[100\left(x_{i+1}-x_{i}^{2}\right)^{2}+\left(x_{i}-1\right)^{2}\right] \\
& R i: f_{3}=\sum_{i=1}^{n}\left(\sum_{j=1}^{i} x_{j}\right)^{2} \\
& \text { Gr: } f_{4}=1+\sum_{i=1}^{n} \frac{x_{i}^{2}}{4000}-\prod_{i=1}^{n} \cos \left(\frac{x_{i}}{\sqrt{i}}\right) \\
& \text { Ra }: f_{5}=10 n+\sum_{i=1}^{n}\left\{x_{i}^{2}-10 \cos \left(2 \pi x_{i}\right)\right\} \\
& \text { Ac: } f_{6}=-20 \exp \left(-0.2 \sqrt{\frac{1}{n} \sum_{i=1}^{n} x_{i}^{2}}\right)-\exp \left(\frac{1}{n} \sum_{i=1}^{n} \cos \left(2 \pi x_{i}\right)\right)+20+e \\
& \text { Sc }: f_{8}=\sum_{i=1}^{n}\left|x_{i}\right|+\prod_{i=1}^{n}\left|x_{i} \sin \left(x_{i}\right)+0.1 x_{i}\right| \\
& L e: f_{7}=\sin ^{2}\left(3 \pi x_{1}\right)+\sum_{i=1}^{n-1}\left(x_{i}-1\right)\left(1+\sin ^{2}\left(3 \pi x_{i+1}\right)\right)+\left(x_{n}-1\right)\left(1+\sin ^{2}\left(2 \pi x_{n}\right)\right)
\end{aligned}
$$

Table 1 shows the characteristics, the terms dependent or independent problem, multipeak, steep denote the dependence relation of the variables, presence of multi-peak and level of steepness, respectively.

Table 1 Characteristics of Benchmark Functions.

\begin{tabular}{|c|c|c|c|c|c|}
\hline Function & Dependent & Multi-peak & Steep & Design range & Global optimum \\
\hline $\mathrm{Sp}$ & No & no & Average & $-5.12 \leq x \leq 5.12$ & $f(0, \ldots, 0)=0$ \\
\hline $\mathrm{Ro}$ & Yes & No & Big & $-2.048 \leq x \leq 2.048$ & $f(1, \ldots, 1)=0$ \\
\hline $\mathrm{Ri}$ & Yes & No & Average & $-51.2 \leq x \leq 51.2$ & $f(0, \ldots, 0)=0$ \\
\hline $\mathrm{Gr}$ & Yes & Yes & Small & $-600.0 \leq x \leq 600.0$ & $f(0, \ldots, 0)=0$ \\
\hline $\mathrm{Ra}$ & No & Yes & Average & $-5.12 \leq x \leq 5.12$ & $f(0, \ldots, 0)=0$ \\
\hline $\mathrm{Ac}$ & No & Yes & Average & $-5.12 \leq x \leq 5.12$ & $f(0, \ldots, 0)=0$ \\
\hline $\mathrm{Le}$ & No & Yes & Average & $-10.0 \leq x \leq 10.0$ & $f(1, \ldots, 1)=0$ \\
\hline $\mathrm{Sc}$ & No & No & Average & $-10.0 \leq x \leq 10.0$ & $f(0, \ldots, 0)=0$ \\
\hline $\mathrm{AL}$ & Yes & Yes & Average & $-10.0 \leq x \leq 10.0$ & $f(0, \ldots, 0)=0$ \\
\hline
\end{tabular}

\subsection{Test to get good value of $\alpha$ in ISADE}

As mention above, we know that the gait of sigmoid function is depend on the sign and value of $\alpha$, Fig. 7 shows the relationship of $F$ and Rank depend on the $\alpha$.

In this section, we test to get best value of $\alpha . N P=8 * D$, maximum iteration iter_max $=$ 3000 , accurate $\varepsilon=10^{-6}, \tau=0.1$ and alpha value $\alpha=-20,-19, \ldots, 19,20$. 
Test to get good value of $\alpha$ factor

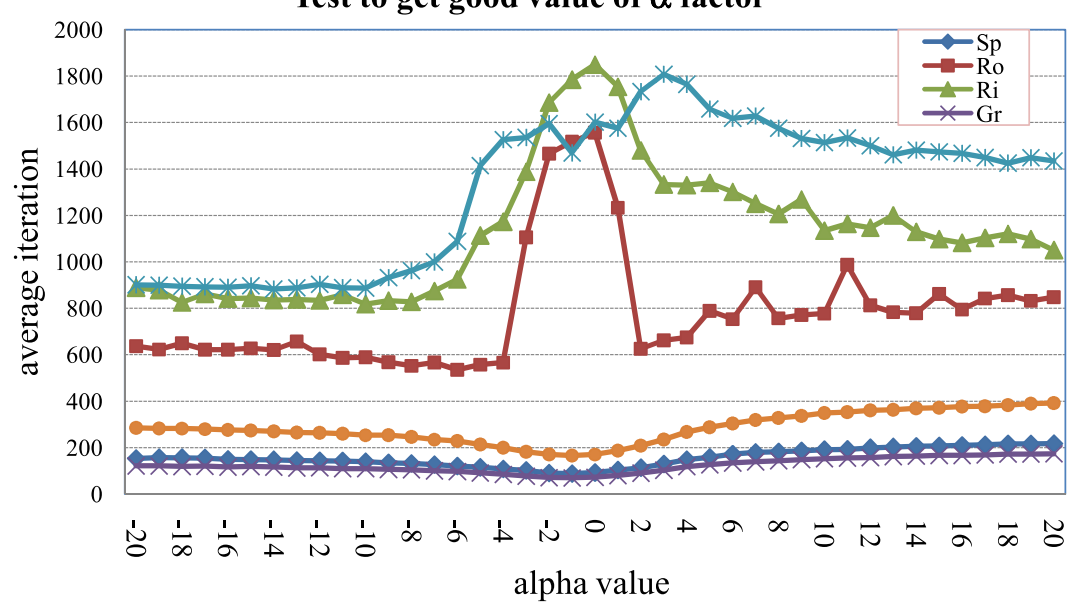

Fig. 7 Result of test to get good value of $\alpha$.

From the result in Fig. 7, the solutions of all the benchmark functions with 30 dimensions reach their global optimum solutions with accurate $\varepsilon=10^{-6}$. However, the result of $\alpha<0$ is better than that of $\alpha>0$. The Sp, Ac, and Gr function are good with $\alpha$ from -10.0 to 10.0 but with Ro, Ri and Ra function is not good at that value, from -10.0 to 20.0 maybe they are not change when we change $\alpha$ value, for best value of all function we will choose $\alpha=-10$ for next test.

\subsection{Test to robust of Algorithm}

4.3.1. ISADE and some approaches are compared in this test with same accurate $\varepsilon=10^{-6}$

Population size was $N P=8 * D$, and accurate $\varepsilon=10^{-6}$ compare the iteration, $\tau=0.1$, at which the optimum is satisfy. The result of this test is shown in table 2 . We present the average of generations and success ratio for the benchmark functions. All the readings given are the averages over 20 independent runs per function.

Table 2 Average of generation and the success ratio

\begin{tabular}{|l|c|c|c|c|c|c|c|c|}
\hline \multirow{2}{*}{ Function } & \multicolumn{2}{|c|}{ PSO } & \multicolumn{2}{c|}{ jDE } & \multicolumn{2}{c|}{ SaDE } & \multicolumn{2}{c|}{ ISADE } \\
\cline { 2 - 9 } & ave_iter & SR & ave_iter & SR & ave_iter & SR & ave_iter & SR \\
\hline Sp & 196.55 & $100 \%$ & 568.90 & $100 \%$ & 238.75 & $100 \%$ & $\mathbf{1 0 7 . 9 0}$ & $100 \%$ \\
\hline Ro & - & $0 \%$ & 5663.15 & $100 \%$ & 1084.06 & $85 \%$ & $\mathbf{7 8 1 . 4 0}$ & $100 \%$ \\
\hline $\mathrm{Ri}$ & 1517.15 & $80 \%$ & 8325.70 & $100 \%$ & 766.95 & $95 \%$ & $\mathbf{4 9 4 . 0 3}$ & $100 \%$ \\
\hline $\mathrm{Gr}$ & 155.30 & $100 \%$ & 457.45 & $100 \%$ & 181.25 & $100 \%$ & $\mathbf{1 2 8 . 9 0}$ & $100 \%$ \\
\hline $\mathrm{Ra}$ & - & $0 \%$ & 3563.65 & $100 \%$ & 1200.8 & $100 \%$ & $\mathbf{1 1 6 4 . 7 5}$ & $100 \%$ \\
\hline $\mathrm{Ac}$ & 390.45 & $100 \%$ & 973.15 & $100 \%$ & 414.50 & $100 \%$ & $\mathbf{3 0 5 . 7 0}$ & $100 \%$ \\
\hline $\mathrm{Le}$ & 298.35 & $100 \%$ & 655.40 & $100 \%$ & $\mathbf{2 6 4 . 0 0}$ & $100 \%$ & 357.30 & $95 \%$ \\
\hline $\mathrm{Sc}$ & 592.85 & $85 \%$ & 996.50 & $100 \%$ & $\mathbf{4 7 3 . 1 0}$ & $100 \%$ & $\mathbf{4 4 9 . 7 0}$ & $100 \%$ \\
\hline $\mathrm{Al}$ & 847.85 & $20 \%$ & - & $0 \%$ & 1898.30 & $100 \%$ & $\mathbf{9 6 6 . 3 0}$ & $100 \%$ \\
\hline
\end{tabular}

As in table 2, ISADE method compare with some reference methods as: $\mathrm{jDE}^{(6)}, \mathrm{PSO}^{(18)}$ and $\mathrm{SaDE}^{(4)}$. We wish to mention here that ISADE is able obtain the optimal solutions for almost benchmark test functions except Le function, with the success ratio equal $100 \%$. The ISADE also gets global optimum at less iteration than that of reference in all benchmark functions, so ISADE could certainly achieve optimal solution with low calculation cost. With four functions $\mathrm{Ro}, \mathrm{Ri}, \mathrm{Ra}$ and $\mathrm{Al}$ the new approach ISADE is very strong robust. The convergence of the optimal solution could be improved more significantly in ISADE than that in EAs for the same accurate.

4.3.2. Test with maximum iteration compares the mean of global minimum and (Std) standard deviation In this experiment, we did test with $N=8 * D, D=30$ dimensions, 
$\tau=0.1$, process will be stop at maximum generation. The mean and standard deviation best fitness of these 20 independent runs has been reported. The result of this test is shown in table 3 .

Table 3 (Mean) Average of global minimum and (std) the standard deviation

\begin{tabular}{|l|c|c|c|c|c|c|c|c|c|}
\hline \multirow{2}{*}{ F } & \multirow{2}{*}{ iter $_{\text {max }}$} & \multicolumn{2}{|c|}{ PSO } & \multicolumn{2}{c|}{ jDE } & \multicolumn{2}{c|}{ SaDE } & \multicolumn{2}{c|}{ ISADE } \\
\cline { 3 - 10 } & & Mean & std & Mean & std & Mean & std & Mean & std \\
\hline $\mathrm{Sp}$ & 500 & $2.83 \mathrm{E}-12$ & $8.24 \mathrm{E}-12$ & $1.14 \mathrm{E}-05$ & $2.77 \mathrm{E}-06$ & $1.32 \mathrm{E}-11$ & $5.77 \mathrm{E}-11$ & $\mathbf{4 . 8 4 E - 3 6}$ & $1.58 \mathrm{E}-35$ \\
\hline $\mathrm{Ro}$ & 1500 & $2.08 \mathrm{E}+01$ & $2.72 \mathrm{E}+00$ & $1.77 \mathrm{E}+01$ & $3.10 \mathrm{E}-01$ & $4.81 \mathrm{E}-18$ & $1.94 \mathrm{E}-17$ & $\mathbf{4 . 4 5 E - 2 8}$ & $1.27 \mathrm{E}-27$ \\
\hline $\mathrm{Ri}$ & 1500 & $6.89 \mathrm{E}-05$ & $5.99 \mathrm{E}-05$ & $1.15 \mathrm{E}+02$ & $3.19 \mathrm{E}+01$ & $2.06 \mathrm{E}-14$ & $6.18 \mathrm{E}-14$ & $\mathbf{3 . 0 6 E}-26$ & $1.19 \mathrm{E}-25$ \\
\hline $\mathrm{Gr}$ & 500 & $1.61 \mathrm{E}-14$ & $3.26 \mathrm{E}-14$ & $2.67 \mathrm{E}-07$ & $7.53 \mathrm{E}-08$ & $\mathbf{2 . 2 2 E - 1 5}$ & $8.00 \mathrm{E}-15$ & $\mathbf{3 . 6 1 E}-16$ & $1.21 \mathrm{E}-16$ \\
\hline $\mathrm{Ra}$ & 1500 & $3.16 \mathrm{E}+01$ & $1.04 \mathrm{E}+01$ & $3.29 \mathrm{E}+01$ & $3.29 \mathrm{E}+00$ & $8.28 \mathrm{E}-12$ & $2.74 \mathrm{E}-11$ & $\mathbf{1 . 3 2 E - 1 4}$ & $5.22 \mathrm{E}-15$ \\
\hline $\mathrm{Ac}$ & 500 & $4.12 \mathrm{E}-07$ & $6.67 \mathrm{E}-07$ & $2.19 \mathrm{E}-03$ & $2.55 \mathrm{E}-04$ & $1.49 \mathrm{E}-07$ & $2.63 \mathrm{E}-07$ & $\mathbf{4 . 0 9 E}-14$ & $1.03 \mathrm{E}-14$ \\
\hline $\mathrm{Le}$ & 500 & $6.12 \mathrm{E}-11$ & $1.56 \mathrm{E}-10$ & $1.75 \mathrm{E}-04$ & $4.84 \mathrm{E}-05$ & $4.04 \mathrm{E}-10$ & $1.40 \mathrm{E}-09$ & $\mathbf{2 . 2 1 E - 3 1}$ & $2.56 \mathrm{E}-31$ \\
\hline $\mathrm{Sc}$ & 500 & $6.69 \mathrm{E}-04$ & $1.43 \mathrm{E}-03$ & $7.95 \mathrm{E}-03$ & $1.14 \mathrm{E}-03$ & $\mathbf{1 . 1 5 E}-06$ & $2.08 \mathrm{E}-06$ & $\mathbf{1 . 2 4 E - 0 6}$ & $2.39 \mathrm{E}-06$ \\
\hline $\mathrm{Al}$ & 1500 & $5.38 \mathrm{E}-12$ & $4.23 \mathrm{E}-12$ & $5.25 \mathrm{E}-03$ & $6.97 \mathrm{E}-04$ & $3.38 \mathrm{E}-05$ & $3.96 \mathrm{E}-05$ & $\mathbf{1 . 0 9 E - 0 9}$ & $3.43 \mathrm{E}-09$ \\
\hline
\end{tabular}

The comparison results are listed in table 3, all of benchmark functions are tested with maximum iteration. From the results, it can be seen that the average of best fitness value and standard deviation of ISADE is better than that of reference $\mathrm{jDE}^{(6)}, \mathrm{PSO}^{(18)}$ and $\mathrm{SaDE}^{(4)}$.

Overall, ISADE was capable of attaining robustness, high quality, low calculation outstanding efficient performance on many benchmark problems. We confirmed satisfactory performance through various benchmark tests.

\subsection{Apply ISADE for constrained engineering optimization}

In this section, we will apply ISADE to solve some real constrained engineering design optimization problems. A set of 4 engineering design optimization problems was chosen to evaluate the performance of our proposed algorithm. A detailed description of the test problems may be consulted in the next subsection. We performed 20 independent runs per problem. our algorithm used the following parameters: $N P=8 * D, \tau=0.1$.

4.4.1. E01: Welded beam design optimization problem The problem is to design a welded beam for minimum cost, subject to some constraints ${ }^{(20)}$. Fig. 8 shows the welded beam structure which consists of a beam $\mathrm{A}$ and the weld required to hold it to member $\mathrm{B}$. The objective is to find the minimum fabrication cost, considering four design variables: $x_{1}, x_{2}$, $x_{3}, x_{4}$ and constraints of shear stress $\tau$, bending stress in the beam $\sigma$, buckling load on the bar $P_{c}$, and end deflection on the beam $\delta$. The optimization model is summarized in the next equation:

Minimize:

$$
f(x)=1.10471 x_{1}^{2} x_{2}+0.04811 x_{3} x_{4}\left(14.0+x_{2}\right)
$$

subject to:

$$
\begin{aligned}
& g_{1}(x)=\tau(x)-13,600 \leq 0 ; \quad g_{2}(x)=\sigma(x)-30,000 \leq 0 \\
& g_{3}(x)=x_{1}-x_{4} \leq 0 ; \quad g_{4}(x)=0.10471 x_{1}^{2}+0.04811 x_{3} x_{4}\left(14.0+x_{2}\right)-5.0 \leq 0 \\
& g_{5}(x)=0.125-x_{1} \leq 0 ; \quad g_{6}(x)=\delta(x)-0.25 \leq 0 ; \quad g_{7}(x)=6,000-P c(x) \leq 0
\end{aligned}
$$

With

$$
\begin{aligned}
& \tau(x)=\sqrt{(\tau,)^{2}+(2 \tau, \tau, \prime) \frac{x_{2}}{2 R}+(\tau,)^{2}} ; \quad \tau=\frac{6,000}{\sqrt{2} x_{1} x_{2}} ; \quad \tau^{\prime \prime}=\frac{M R}{J} \\
& M=6,000\left(14.0+\frac{x_{2}}{2}\right) ; \quad R=\sqrt{\frac{x_{2}^{2}}{4}+\left(\frac{x_{1}+x_{3}}{2}\right)^{2}} ; \quad J=2\left\{x 1 x 2 \sqrt{2}\left[\frac{x_{2}^{2}}{4.0}+\left(\frac{x_{1}+x_{3}}{2}\right)^{2}\right]\right\} \\
& \sigma(x)=\frac{504,000}{x_{4} x_{3}^{2}} ; \quad \delta(x)=\frac{65,856,000}{\left(30 \times 10^{6}\right) x_{4} x_{3}^{3}} \\
& P c(x)=\frac{4.013\left(30 \times 10^{6}\right) \sqrt{\frac{x_{3}^{2} x_{4}^{6}}{36.0}}}{196.0}\left(1-\frac{x_{3} \sqrt{\frac{30 \times 10^{6}}{4\left(12 \times 10^{6}\right)}}}{28.0}\right)
\end{aligned}
$$

With $0.1 \leq x_{1}, x_{4} \leq 2.0$, and $0.1 \leq x_{2}, x_{3} \leq 10.0$. 
Where $f\left(x^{*}\right)=1.724852$

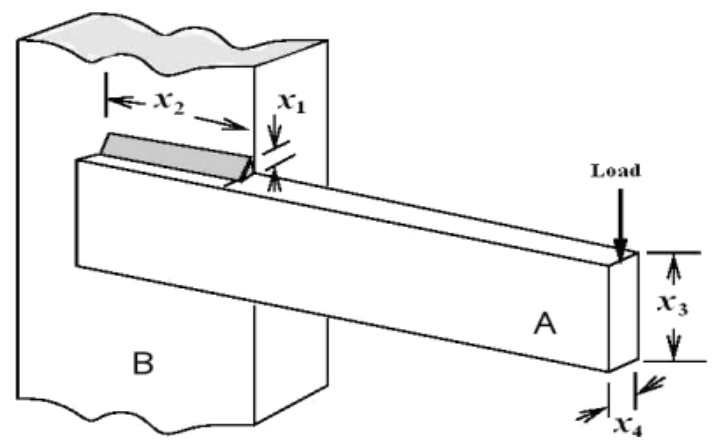

Fig. 8 Welded Beam.

4.4.2. E02: Pressure vessel design optimization problem A compressed air storage tank with a working pressure of 3,000 psi and a minimum volume of $750 \mathrm{ft}^{3}$. A cylindrical vessel is capped at both ends by hemispherical heads (see Fig. 9). Using rolled steel plate, the shell is made in two halves that are joined by toe longitudinal welds to form a cylinder. The objective is minimize the total cost, including the cost of the materials forming the welding ${ }^{(21)}$. The design variables are: thickness $x_{1}$, thickness of the head $x_{2}$, the inner radius $x_{3}$, and the length of the cylindrical section of the vessel $x_{4}$. The variables $x_{1}$ and $x_{2}$ are discrete values which are integer multiples of 0.0625 inch. Then, The mathematical formulation of this problem is: Minimize:

$$
f(x)=0.6224 x_{1} x_{3} x_{4}+1.7781 x_{2} x_{3}^{2}+3.1661 x_{1}^{2} x_{4}+19.84 x_{1}^{2} x_{3}
$$

subject to:

$$
\begin{array}{ll}
g_{1}(x)=-x_{1}+0.0193 x_{3} \leq 0 ; & g_{2}(x)=-x_{2}+0.00954 x_{3} \\
g_{3}(x)=-\pi x_{3}^{2} x_{4}-\frac{4}{3} \pi x_{3}^{3}+1,296,000.0 \leq 0 ; & g_{4}(x)=x_{4}-240.0 \leq 0
\end{array}
$$

With $1 \times 0.0625 \leq x_{1}, x_{2} \leq 99 \times 0.0625,10.0 \leq x_{3}$, and $x_{4} \leq 200.0$.

Best solution: $x^{*}=(0.8125,0.4375,42.098446,176.636596)$

Where $f\left(x^{*}\right)=6,059.714335$.

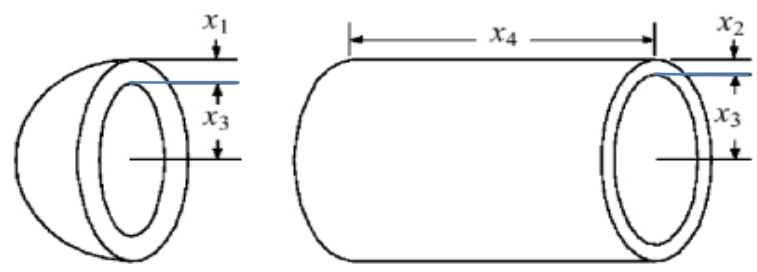

Fig. 9 Pressure Vessel.

4.4.3. E03: Speed reducer design optimization problem The design of the speed reducer $^{(22)}$ shown in Fig. 10, is considered with the face width $x_{1}$, module of teeth $x_{2}$, number of teeth on pinion $x_{3}$, length of the first shaft between bearings $x_{4}$, length of the second shaft between bearings $x_{5}$, diameter of the first shaft $x_{6}$, and diameter of the first shaft $x_{7}$ (all variables continuous except $x_{3}$ that is integer). The weight of the speed reducer is to be minimized subject to constraints on bending stress of the gear teeth, surface stress, transverse deflections of the shafts and stresses in the shaft. The mathematical formulation of this problem is: Minimize:

$$
\begin{aligned}
f(x)= & 0.7854 x_{1} x_{2}^{2}\left(3.3333 x_{3}^{2}+14.9334 x_{3}-43.0934\right)-1.508 x_{1}\left(x_{6}^{2}+x_{7}^{2}\right) \\
& +7.4777\left(x_{6}^{3}+x_{7}^{3}\right)+0.7854\left(x_{4} x_{6}^{2}+x_{5} x_{7}^{2}\right)
\end{aligned}
$$


subject to:

$$
\begin{aligned}
& g_{1}(x)=\frac{27.0}{x_{1} x_{2}^{2} x_{3}}-1.0 \leq 0 ; \quad g_{2}(x)=\frac{397.5}{x_{1} x_{2}^{2} x_{3}^{2}}-1.0 \leq 0 \\
& g_{3}(x)=\frac{1.93 x_{4}^{3}}{x_{2} x_{3} x_{6}^{2}}-1.0 \leq 0 ; \quad g_{4}(x)=\frac{1.93 x_{5}^{3}}{x_{2} x_{3} x_{7}^{4}}-1.0 \leq 0 \\
& g_{5}(x)=\frac{1.0}{110.0 x_{6}^{3}} \sqrt{\left(\frac{745.0 x_{4}}{x_{2} x_{3}}\right)^{2}+16.9 \times 10^{6}-1.0 \leq 0} \\
& g_{6}(x)=\frac{1.0}{85.0 x_{7}^{3}} \sqrt{\left(\frac{745.0 x_{5}}{x_{2} x_{3}}\right)^{2}+157.5 \times 10^{6}}-1.0 \leq 0 \\
& g_{7}(x)=\frac{x_{2} x_{3}}{40.0}-1.0 \leq 0 ; \quad g_{8}(x)=\frac{5.0 x_{2}}{x_{1}}-1.0 \leq 0 ; \quad g_{9}(x)=\frac{x_{1}}{12.0 x_{2}}-1.0 \leq 0 \\
& g_{10}(x)=\frac{1.5 x_{6}+1.9}{x_{4}}-1.0 \leq 0 ; \quad g_{11}(x)=\frac{1.1 x_{7}+1.9}{x_{5}}-1.0 \leq 0
\end{aligned}
$$

With $2.6 \leq x_{1} \leq 3.6,0.7 \leq x_{2} \leq 0.8,17 \leq x_{3} \leq 28,7.3 \leq x_{4} \leq 8.3,7.8 \leq x_{5} \leq 8.3$, $2.9 \leq x_{6} \leq 3.9$, and $5.0 \leq x_{7} \leq 5.5$,

Best solution: $x^{*}=(3.500000,0.7,17,7.300000,7.800000,3.350214,5.286683)$

Where $f\left(x^{*}\right)=2,996.348165$.

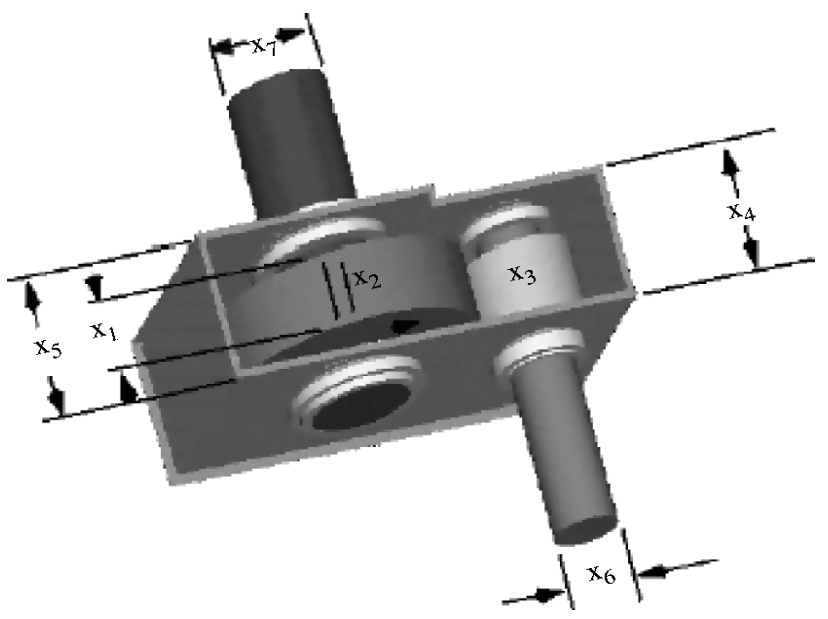

Fig. 10 Speed Reducer.

4.4.4. E04: Tension/compression spring design optimization problem This problem (23), (24) minimizes the weight of a tension/compression spring Fig. 11, subject to constraints of minimum deflections, shear stress, surge frequency, limits on outside diameter and on design variables. There are three design variables: the wire diameter $x_{1}$, the mean coil diameter $x_{2}$, and the number of active coils $x_{3}$. The mathematical formulation of this problem is: Minimize:

$$
f(x)=\left(x_{3}+2\right) x_{2} x_{1}^{2}
$$

subject to:

$$
\begin{aligned}
& g_{1}(x)=1.0-\frac{x_{2}^{3} x_{3}}{7,1785 x_{1}^{4}} \leq 0 ; \\
& g_{3}(x)=1.0-\frac{140.45 x_{1}}{x_{2}^{2} x_{3}} \leq 0 \text {; } \\
& g_{2}(x)=\frac{4.0 x_{2}^{2}-x_{1} x_{2}}{12,566\left(x_{2} x_{1}^{3}-x_{1}^{4}\right)}+\frac{1.0}{5,108 x_{1}^{2}}-1.0 \leq 0 \\
& g_{4}(x)=\frac{x_{1}+x_{2}}{1.5}-1.0 \leq 0
\end{aligned}
$$

With $0.05 \leq x_{1} \leq 2.0,0.25 \leq x_{2} \leq 1.3$, and $2.0 \leq x_{3} \leq 15.0$.

Best solution: $x^{*}=(0.051690,0.356750,11.287126)$

Where $f\left(x^{*}\right)=0.012665$.

4.4.5. Result of applying ISADE for constrained engineering optimization Table 4 and 5 show the vectors of the best solution as well as the values of the constraint terms reached by ISADE, for each of the problems tested. We got the optimum solution at 8000 objective function evaluations (FE) per run with the welded beam problem, 14000 with the pressure vessel problem, 15000 with the speed reducer problem and 8000 with the tension/compression spring problem. We also tested the algorithm with more than above of objective function evaluation, but no performance improvements. 


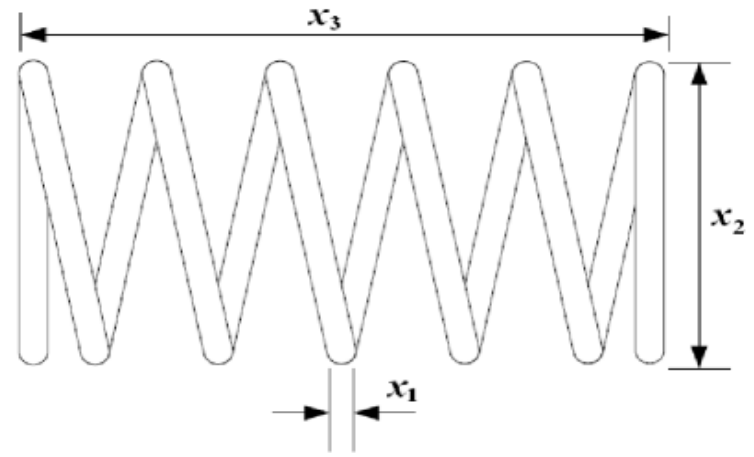

Fig. 11 Tension/Compression Spring.

Table 4 Result of applying ISADE for E01 and E02 problem.

\begin{tabular}{|c|c|c|c|c|c|c|c|}
\hline \multicolumn{4}{|c|}{ E01 (Welded beam) } & \multicolumn{4}{c|}{ E02 (Pressure vessel } \\
\hline \multicolumn{2}{|c|}{ Solutions } & \multicolumn{2}{|c|}{ Constraints } & \multicolumn{2}{|c|}{ Solutions } & \multicolumn{2}{c|}{ Constraints } \\
\hline$x_{1}$ & 0.205730 & $g_{1}(x)$ & $-4.60 \mathrm{E}-07$ & $x_{1}$ & 0.812500 & $g_{1}(x)$ & 0.0 \\
\hline$x_{2}$ & 3.470489 & $g_{2}(x)$ & $-1.20 \mathrm{E}-06$ & $x_{2}$ & 0.437500 & $g_{2}(x)$ & $-3.59 \mathrm{E}-02$ \\
\hline$x_{3}$ & 9.036624 & $g_{3}(x)$ & $-3.97 \mathrm{E}-12$ & $x_{3}$ & 42.098446 & $g_{3}(x)$ & 0.0 \\
\hline$x_{4}$ & 0.205730 & $g_{4}(x)$ & $-3.97 \mathrm{E}-12$ & $x_{4}$ & 176.636596 & $g_{4}(x)$ & $-6.34 \mathrm{E}+01$ \\
\hline $\mathrm{f}(\mathrm{x})$ & $\mathbf{1 . 7 2 4 8 5 2}$ & $g_{5}(x)$ & $-3.97 \mathrm{E}-12$ & $\mathrm{f}(\mathrm{x})$ & $\mathbf{6 0 5 9 . 7 1 4}$ & & \\
\hline $\mathrm{FE}$ & 8000 & $g_{6}(x)$ & $-3.97 \mathrm{E}-12$ & FE & 24000 & & \\
\hline & & $g_{7}(x)$ & $-3.43 \mathrm{E}+00$ & & & & \\
\hline
\end{tabular}

Table 5 Result of applying ISADE for E03 and E04 problem.

\begin{tabular}{|c|c|c|c|c|c|c|c|c|c|}
\hline \multicolumn{2}{|c|}{ E04 (Tension/Compression spring) } & \multicolumn{6}{c|}{ E03 (Speed reducer) } \\
\hline \multicolumn{2}{|c|}{ Solutions } & \multicolumn{2}{c|}{ Constraints } & \multicolumn{2}{c|}{ Solutions } & \multicolumn{2}{c|}{ Constraints } & \multicolumn{2}{c|}{ Constraints } \\
\hline$x_{1}$ & 0.051690 & $g_{1}(x)$ & 0.0 & $x_{1}$ & 3.500008 & $g_{1}(x)$ & $-7.39 \mathrm{E}-02$ & $g_{9}(x)$ & $-5.83 \mathrm{E}-01$ \\
\hline$x_{2}$ & 0.356742 & $g_{2}(x)$ & 0.0 & $x_{2}$ & 0.700000 & $g_{2}(x)$ & $-1.98 \mathrm{E}-01$ & $g_{10}(x)$ & $-5.13 \mathrm{E}-02$ \\
\hline$x_{3}$ & 11.287534 & $g_{3}(x)$ & -4.05 & $x_{3}$ & 17.000014 & $g_{3}(x)$ & $-4.99 \mathrm{E}-01$ & $g_{11}(x)$ & $-1.09 \mathrm{E}-02$ \\
\hline$f(x)$ & $\mathbf{0 . 0 1 2 6 6 5}$ & $g_{4}(x)$ & $-7.28 \mathrm{E}-01$ & $x_{4}$ & 7.300133 & $g_{4}(x)$ & $-9.01 \mathrm{E}-01$ & F-Call & 15000 \\
\hline FE & 8000 & & & $x_{5}$ & 7.8000016 & $g_{5}(x)$ & $-9.16 \mathrm{E}-06$ & & \\
\hline & & & & $x_{6}$ & 3.350225 & $g_{6}(x)$ & $-3.08 \mathrm{E}-07$ & & \\
\hline & & & & $x_{7}$ & 5.286684 & $g_{7}(x)$ & $-7.02 \mathrm{E}-01$ & & \\
\hline & & & & $f(x)$ & $\mathbf{2 9 9 6 . 3 5 8}$ & $g_{8}(x)$ & $-2.30 \mathrm{E}-06$ & & \\
\hline
\end{tabular}

\section{Conclusion}

Locating global minimizers is a very challenging task for any minimization method. In this research, a new improvement of self-adaptive differential evolution is proposed. The main idea is that the three mutation scheme operators are chosen to be applied to individuals in the current population with the same probability, the scalar factor $F$ is adaptively calculated by sigmoid function after ranking population in their fitness value and the control parameter $C R$ is adjusted to balance the abilities of DE in exploitation and DE in exploration.

Some benchmark test functions and constrained engineering optimization are used to validate the performance of the ISADE. The proposed approach performed well in several test problems both in terms of the number of fitness function evaluations required and in terms of the quality of the solutions found. The results show that ISADE outperforms in most function minimization. The results are also proved that the convergence speed of ISADE is faster that of $\mathrm{jDE}^{(6)}, \mathrm{PSO}^{(18)}$ and $\mathrm{SaDE}^{(4)}$.

\section{References}

( 1 ) Storn, R., and Price, K., Differential Evolution - A Simple and Efficient Adaptive Scheme for Global Optimization over Continuous Spaces, Technical report tr-95-012, ICSI (1995). 
( 2 ) Storn, R., and Price, K., Differential Evolution-A Simple and Efficient Heuristic for Global Optimization over Continuous Spaces, Journal Global Optimization, Vol. 11 (1997), pp. 341-359.

( 3 ) S.Soliman, O., and T. Bui, L., A Self-Adaptive Strategy for Controlling Parameters in Differential Evolution, Evolutionary Computation, IEEE World Congress on Computational Intelligence, pp. 2837-2842 (2008).

( 4 ) K.Qin, A., and N.Suganthan, P., Self-Adaptive Differential Evolution Algorithm for Numerical Optimization, Evolutionary Computation, IEEE Congress (CEC2005), Vol. 2 (2005), pp. 1785-1791.

( 5 ) Greiner, S., Bokovic, B., Mernik, M., Brest, J., and Zumer, V., Self-Adapting Control Parameters in Differential Evolution: A Comparative Study on Numerical Benchmark Problems, IEEE Transactions On Evolutionary Computation, Vol. 10, No. 6 (2006), pp. 646-657.

( 6 ) Greiner, S., Bokovic, B., Mernik, M., Brest, J., and Zumer, V., Performance Comparison of Self-Adaptive and Adaptive Differential Evolution Algorithms, Soft Comput, Vol. 11, No. 7 (2007), pp. 617-629.

( 7 ) Deb, K., and B. Agrawal, R., Simulated Binary Crossover for Continuous Search Space, Complex Systems, vol. 9 (1995), pp. 115-148.

( 8 ) Eiben, A., Hinterding, R., and Michalewicz, Z., Parameter Control in Evolutionary Algorithms, Evolutionary Computation, IEEE Transactions, Vol. 3, No. 2 (1999-7), pp. 124-141.

( 9 ) J.Fogel, L., J.Angeline, P., and B.Fogel, D., An Evolutionary Programming Approach to Self-adaptation in Finite State Machines, Proceeding the of Fourth Annual Conference on Evolutionary Programming, Cambridge, MA, pp. 355-365 (1995).

(10) S.Soliman, O,. T.Bui, L, and A. Abbass, H., The Effect of a Stochastic Step Length on the Performance of the Differential Evolution Algorithm, Evolutionary Computation, IEEE Congress (CEC2007), pp. 2850-2857 (2007).

(11) Liu, J,. and Lampinen, J., Fuzzy Adaptive Differential Evolution Algorithm, Proceedings of the IEEE region 10 conference on computer, communications, control and power engineering, Vol. 1 (2002), pp. 606-611.

(12) Liu, J,. and Lampinen, J., A Fuzzy Adaptive Differential Evolution Algorithm, Soft Computing - A Fusion of Foundations, Methodologies and Applications, Vol. 9, No. 6 (2005), pp.448-462.

(13) V. Price, K., An Introduction to Differential Evolution, New ideas in Optimization, McGraw-Hill Ltd., UK Maidenhead, UK, England, pp. 79-108 (1999).

(14) A.Abbass, H., The Self-Adaptive Pareto Differential Evolution Algorithm, Proceedings of the IEEE Congress on Evolutionary Computation(CEC2002), Vol. 1, pp. 831-836 (2002).

(15) Meza, R,. Sanchis, G,. Blasco, J,. and Herrero, X., Hybrid DE Algorithm with Adaptive Crossover Operator for Solving Real-world Numerical Optimization Problems, Evolutionary Computation (CEC2011), IEEE Congress, pp.1551-1556 (2011).

(16) Teo, J., Exploring Dynamic Self-adaptive Populations in Differential Evolution, Soft Comput, Vol. 10, No. 8 (2006), pp.673-686.

(17) Hasegawa, H., and Tooyama, S., Adaptive Plan System with Genetic Algorithm using the Variable Neighborhood range Control, Evolutionary Computation (CEC 2009), IEEE Congress, pp. 846-853 (2009).

(18) Kennedy, J., and Eberhart, R., Particle Swarm Optimization, Neural Networks, Proceedings of IEEE International Conference, Vol. 4 (1995), pp. 1942-1948.

(19) Karaboga, D., An Idea Based On Honey Bee Swarm for Nunberical Optimization, Technical Report-Tr-06, October, 2005.

(20) Ragsdell, K,. and Phillips, D., Optimal Design of a Class of Welded Structures using Geometric Programming, J. Eng. Ind, Vol. 98, No. 3 (1976), pp. 1021-1025. 
(21) Sandgren, E., Nonlinear Integer and Discrete Pro-gramming in Mechanical Design Optimization, J.Mech. Des.-T. ASME, Vol. 112, No. 2 (1990), pp. 223-229.

(22) Golinski, J., An Adaptive Optimization System Applied to Machine Synthesis, Mech. Mach. Theory, Vol. 8, No. 4 (1973), pp. 419- 436.

(23) Arora, J., Introduction to Optimum Design, McGrawHill, 1989.

(24) Belegundu, A., A Study of Mathematical Programming Methods for Structural Optimization, PhD thesis, Department of Civil Environmental Engineering, University of Iowa, Iowa, 1982. 